\title{
SYNTHESIS, SPECTRAL ANALYSIS AND EVOLUTION OF ANTIMICROBIAL ACTIVITY OF SCHIFF BASE OF CYANOACETOHYDRAZIDE
}

\author{
Juvansinh Jadeja, Mayank Mamtora, Mitesh Gondaliya, Rohit Manawar, \\ Manish K. Shah* \\ Chemical Research Laboratory, Department of Chemistry \\ Saurashtra University, Rajkot - 360005 \\ E-mail address: drmkshah99@gmail.com
}

Keywords: Schiff's base; Spectral analysis; Antimicrobial activity; Cyanoacetohydrazide; Aldehyde

\begin{abstract}
In search of some antimicrobial agents, synthesis of some Schiff base of cyanoacetohydrazide derivatives has been reported. The structures of all the synthesized compounds were established on the bases of various spectroscopic methods. All Schiff base were screened for antimicrobial activity. cyanoacetohydrazide were found to possess better antimicrobial potential.
\end{abstract}

\section{INTRODUCTION}

Hydrazide-hydrazones and their derivatives are important class of compounds which is use in organic synthesis[1,2]. As antibiotic resistant organisms have become more common place, the pipeline for the discovery of new antimicrobial agents has decreased[3]. The pharmacological profiles are their antimicrobial [4,5], anti-tubercular [6,7], anticonvulsant [8,9] anti-inflammatory $[10,11]$, antidepressant [12], antitumor [13] and analgesic activities [14]. Few hydrazones act as orally effective drugs for the treatment of iron overload disease or genetic disease-thalassemic[15]. We focused our work on developing novel polyfunctionalized heterocyclic compounds with potential bioactivity.

\section{EXPERIMENTAL}

\subsection{Material and method}

All the compounds and solvents were purchased from spectrochem and lobachemie, and checked TLC and Melting points were determined by open capillary tubing and are uncorrected. IR spectra were taken with Shimadzu IR Affinity-1S FTIR spectrometer. Mass spectra were done on GCMS QP2010 mass spectrometer, 1H NMR spectra were taken on Bruker NMR spectrometer (400 MHz), using TMS [as internal standard]. Elemental data was recorded by Carlo Erba EA 1108 elemental analyzer.

\subsubsection{General procedure for the synthesis of 2-cyanoacetohydrazide}

Cyanoacetichydrazide was synthesized according to (Bondock et al., 2006) procedure. Hydrazine hydrate $(0.1 \mathrm{~mol})$ and ethyl cyanoacetate $(0.1 \mathrm{~mol})$ was added in $20 \mathrm{ml}$ ethanol as solvent in FBF at cooling condition and stirred it till it becomes solution. White precipitate was formed in 10-15 min. It was than Filtered and washed with ethanol or Ether and dried. Yield: 95\%, m.p. $=109^{\circ} \mathrm{C}$.

\subsubsection{Synthesis of Schiff base of 2-cyanoacetohydrazide}

Synthesis of Schiff base of 2-cyanoacetohydrazide with different heterocyclic aldehyde was prepared by adding equimolar amounts of 2-cyanoacetohydrazide and different aldehyde in 50 $\mathrm{mL}$ absolute ethanol and was added 2-3 drops of glacial acetic acid respectively. The resulting mixture was magnetically stirred while cold for an hour. The reaction was examine by TLC using hexane:ethyl acetate(2:3) system. The precipitated products was filtered and crystallized from ethanol and dried by vacuum for 1 hour. 


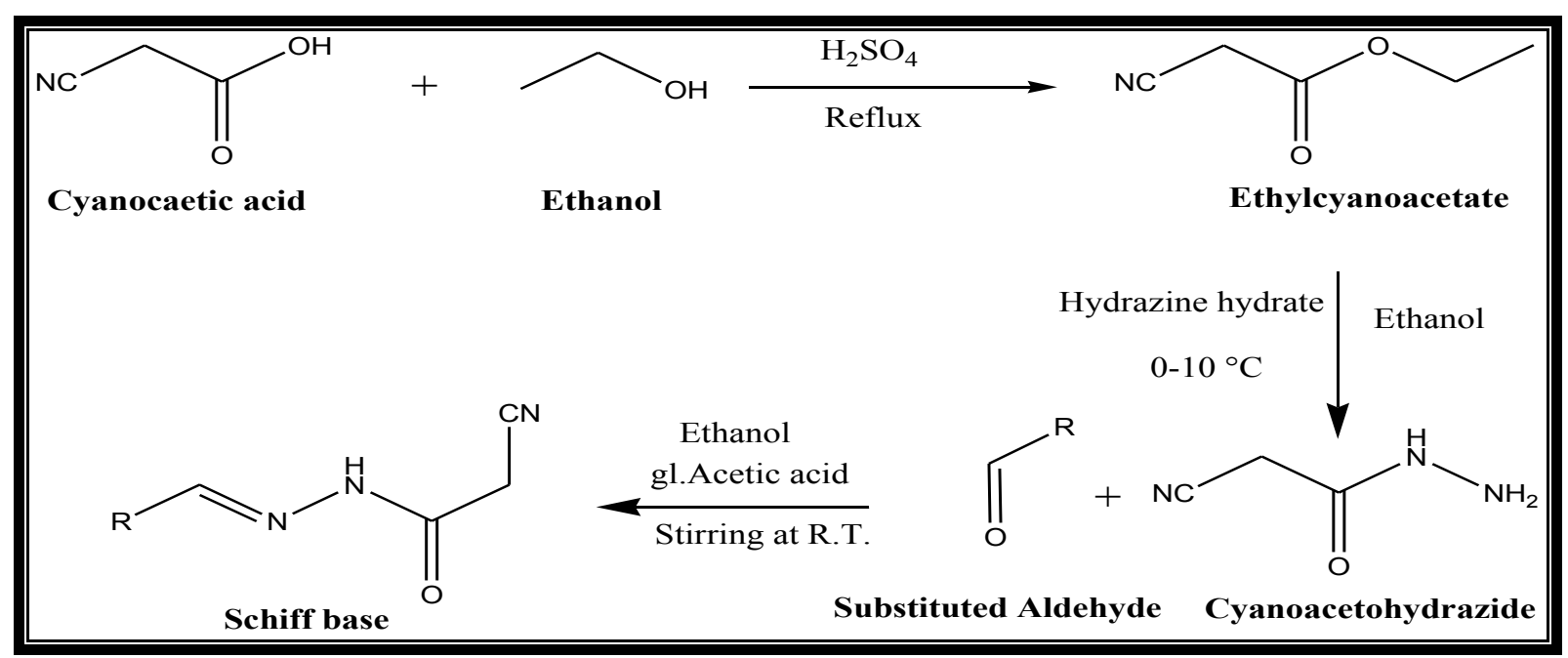

Figure. 1 Reaction Scheme

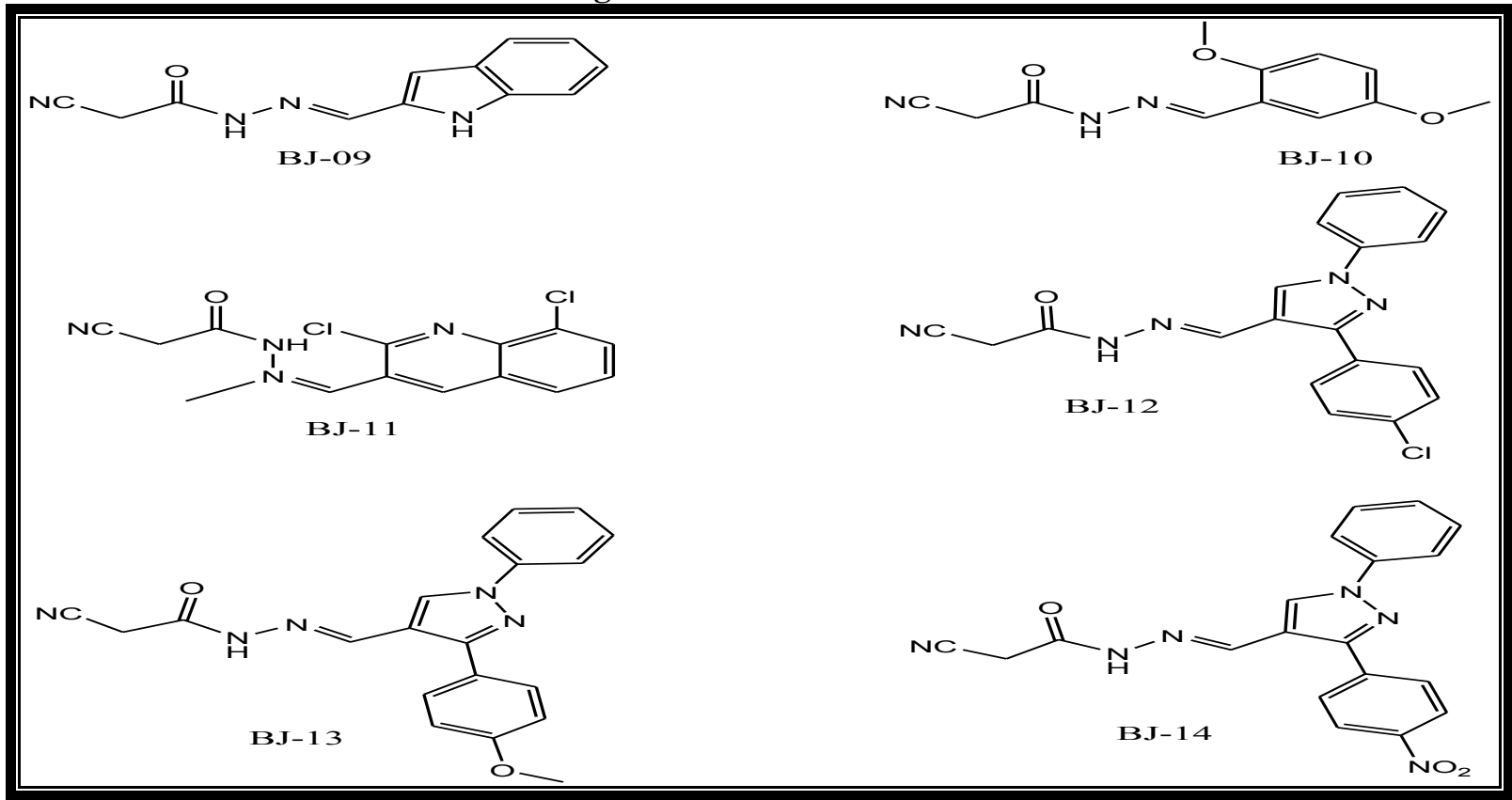

Figure. 2 Structure of Schiff Base

\section{RESULT AND DISSCUTION}

\subsection{Spectral Analysis}

3.1.1 N'-((1H-indol-2-yl)methylene)-2-cyanoacetohydrazide [BJ-09]

Color- Light Yellow, Yield- 85\%, Melting Point- $215^{\circ} \mathrm{C}$, Elemental Analytical Calculation for $\boldsymbol{C}_{12} \mathrm{H}_{10} \mathrm{~N}_{4} \mathrm{O}(226.09 \mathrm{~g} / \mathrm{mol}): \mathrm{C}, 63.71 \% ; \mathrm{H}, 4.46 \%$; N, 24.76\%; O, 7.07\%. Found: C, 63.60\%; H, 4.40 5\%; N, 24.73\%; O, 7.02\%; MS (m/z): 226; IR $\left(\mathbf{c m}^{-1}\right): v(\mathrm{NH}) 3290,3088 ; v(\mathrm{C}=\mathrm{N})$ 1666; $v(\mathrm{C}=\mathrm{O}) 1716 ; \quad v(\mathrm{~N}-\mathrm{N}) 1107 ; \mathrm{v}(\mathrm{Ar}-\mathrm{C}-\mathrm{H})$ 2953; $v(\mathrm{Ar}-\mathrm{C}=\mathrm{C})$ 1539; $v(\mathrm{C}=\mathrm{N})$ 2276, 2324; ${ }^{\boldsymbol{H}} \boldsymbol{H}-\boldsymbol{N M} \boldsymbol{R}$ (DMSO-d $\left.)_{6}\right): \delta$ ppm $4.23\left(\mathrm{~s}, 2 \mathrm{H}, \mathrm{CH}_{2}\right) ; 8.20,(\mathrm{~s}, 1 \mathrm{H}, \mathrm{Ar}-\mathrm{H}) ; 8.12-8.14(\mathrm{~d}, 1 \mathrm{H}, \mathrm{Ar}-\mathrm{H}) ; 7.81-7.85,(\mathrm{~m}$, 1H,Ar-H);7.16-7.21, (m, 1H, Ar-H); 7.43-7.45, (m, 1H, Ar-H); 8.34 (s, 1H, HC=N); 11.50 (s, 1H, NH), 11.61 (s, 1H, NH). ${ }^{13} \boldsymbol{C}$-NMR (DMSO-d $): \delta$ ppm 24.58( $\left(\mathrm{CH}_{2}\right) ; 116.47(\mathrm{CN}), 111.20$, 111.94, 120.76, 121.97, 122.79, 124.00, $(\mathrm{C}=\mathrm{C}), 131.06,137.14$ (fused $\mathrm{C}=\mathrm{C})(\mathrm{Ph}) ; 142.06(\mathrm{HC}=\mathrm{N})$; $163.91(\mathrm{C}=\mathrm{O})$.

\subsubsection{N'-(2,5-dimethoxybenzylidene)-2-cyanoacetohydrazide [BJ-10]}

Color-White, Yield- 80\%, Melting Point- $205^{\circ} \mathrm{C}$, Elemental Analytical Calculation for $\boldsymbol{C}_{12} \mathrm{H}_{13} \mathrm{~N}_{3} \boldsymbol{O}_{3}(247.25 \mathrm{~g} / \mathrm{mol}): \mathrm{C}, 58.29 \%$; H, 5.30\%; N, 16.99\%; O, $19.41 \%$. Found: C, 58.25\%; H, 5.28\%; N, 16.95\%; O, 19.40\%; MS (m/z): 247; IR $\left(\mathrm{cm}^{-1}\right): v(\mathrm{NH}) 2999,3088 ; \quad v(\mathrm{C}=\mathrm{N}) 1683$; $v(\mathrm{C}=\mathrm{O}) 1716 ; \mathrm{v}(\mathrm{N}-\mathrm{N})$ 1103; $v(\mathrm{Ar}-\mathrm{C}-\mathrm{H}) 2837,2902 ; \mathrm{v}(\mathrm{Ar}-\mathrm{C}=\mathrm{C})$ 1558; $(\mathrm{C}=\mathrm{N}) 2357,2328 ; \mathrm{v}(\mathrm{C}-\mathrm{O})$ 
1045. ${ }^{1} \boldsymbol{H}$-NMR (DMSO-d $\left.)_{6}\right): \delta$ ppm 3.74-3.78 (s, 6H, CH $) ; 4.22\left(\mathrm{~s}, 2 \mathrm{H}, \mathrm{CH}_{2}\right) ; 7.28(\mathrm{~s}, 1 \mathrm{H}, \mathrm{Ar}-\mathrm{H})$; $7.01-7.02(\mathrm{~d}, 1 \mathrm{H}, \mathrm{Ar}-\mathrm{H}) ; 7.04-7.10,(\mathrm{~d}, 1 \mathrm{H}, \mathrm{Ar}-\mathrm{H}) ; 8.30(\mathrm{~s}, 1 \mathrm{H}, \mathrm{HC}=\mathrm{N}) ; 11.77(\mathrm{~s}, 1 \mathrm{H}, \mathrm{NH}) .{ }^{13} \boldsymbol{C}-$ NMR (DMSO-d 6 ): $\delta$ ppm 55.36, $56.04\left(\mathrm{OCH}_{3}\right) ; 24.31\left(\mathrm{CH}_{2}\right) ; 116.09(\mathrm{CN}), 122.22,117.20,113.06$, $109.72(\mathrm{C}=\mathrm{C}) ; 139.64(\mathrm{HC}=\mathrm{N}) ; 153.07,152.04(\mathrm{C}-\mathrm{O}) ; 164.67(\mathrm{C}=\mathrm{O})$.

\subsubsection{N'-((2,8-dichloroquinolin-3-yl)methylene)-2-cyanoacetohydrazide [BJ-11]}

Color-crimish, Yield- $88 \%$, Melting Point- $215^{\circ} \mathrm{C}$, Elemental Analytical Calculation for $\mathrm{C}_{13} \mathrm{H}_{8} \mathrm{~N}_{4} \mathrm{OCl}(306.01 \mathrm{~g} / \mathrm{mol}): \mathrm{C}, 50.84 \% ; \mathrm{H}, 2.63 \%$; N, 18.24\%; O, 5.21\%; Cl, 23.09 \%. Found: C,

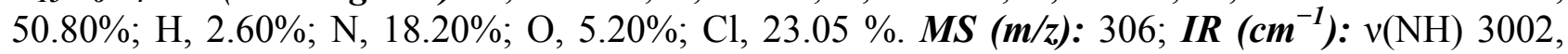
3080; $v(\mathrm{C}=\mathrm{N}) 1680 ; v(\mathrm{C}=\mathrm{O}) 1718 ; \mathrm{v}(\mathrm{N}-\mathrm{N}) 1100 ; \mathrm{v}(\mathrm{Ar}-\mathrm{C}-\mathrm{H}) 2830,2912 ; \mathrm{v}(\mathrm{Ar}-\mathrm{C}=\mathrm{C}) 1560 ; v(\mathrm{C}=\mathrm{N})$ 2340, 2330. ${ }^{1} \boldsymbol{H}$-NMR (DMSO-d $)$ ): $\delta$ ppm $4.20\left(\mathrm{~s}, 2 \mathrm{H}, \mathrm{CH}_{2}\right) ; 7.02-7.04(\mathrm{~d}, 1 \mathrm{H}, \mathrm{Ar}-\mathrm{H}) ; 7.09-7.12$ $(\mathrm{d}, 1 \mathrm{H}, \mathrm{Ar}-\mathrm{H}) ; 6.90-6.98(\mathrm{~m}, 1 \mathrm{H}, \mathrm{Ar}-\mathrm{H}) ; 8.80(\mathrm{~s}, 1 \mathrm{H}, \mathrm{Ar}-\mathrm{H}) ; 8.50(\mathrm{~s}, 1 \mathrm{H}, \mathrm{HC}=\mathrm{N}) ; 11.57(\mathrm{~s}, 1 \mathrm{H}$, NH). ${ }^{13} \boldsymbol{C}$-NMR (DMSO-d $)$ : $\delta$ ppm $24.20\left(\mathrm{CH}_{2}\right) ; 116.06(\mathrm{CN}), 125.22,127.20,135.06,138.72$, $138.50(\mathrm{C}=\mathrm{C}) ; 142.40,143.64(\mathrm{HC}=\mathrm{Cl}) ; 140.64(\mathrm{HC}=\mathrm{N}) ; 153.07,152.04(\mathrm{C}-\mathrm{O}) ; 168.86(\mathrm{C}=\mathrm{O})$.

3.1.4 N'-((3-(4-chlorophenyl)-1-phenyl-1H-pyrazol-4-yl)methylene)-2-cyanoacetohydrazide [BJ-12]

Color-yellow, Yield- 70\%, Melting Point- $230^{\circ} \mathrm{C}$, Elemental Analytical Calculation for $\boldsymbol{C}_{19} \mathrm{H}_{14} \mathrm{~N}_{5} \mathrm{OCl}(363.08 \mathrm{~g} / \mathrm{mol}): \mathrm{C}, 62.73 \% ; \mathrm{H}, 3.88 \%$; N, 19.75\%; O, $4.40 \%$; Cl 9.75\%. Found: C, 62.70\%; H, 3.84\%; N, 19.65\%; O, 4.38\%; Cl 9.70\%. MS (m/z): 363; IR (cm $\left.{ }^{-1}\right): v(\mathrm{NH}) 3005,3088$; $v(\mathrm{C}=\mathrm{N}) 1680 ; v(\mathrm{C}=\mathrm{O}) 1718 ; \mathrm{v}(\mathrm{N}-\mathrm{N}) 1103,1088,1050 ; \mathrm{v}(\mathrm{Ar}-\mathrm{C}-\mathrm{H}) 2840,2950 ; \mathrm{v}(\mathrm{Ar}-\mathrm{C}=\mathrm{C}) 1580$; $v(\mathrm{C}=\mathrm{N}) 2380,2228 .{ }^{1} \boldsymbol{H}$-NMR (DMSO-d $)$ ): $\delta$ ppm $4.22\left(\mathrm{~s}, 2 \mathrm{H}, \mathrm{CH}_{2}\right) ; 7.28-7.34(\mathrm{~m}, 7 \mathrm{H}, \mathrm{Ar}-\mathrm{H})$; 7.42-7.44 (d, 2H, Ar-H); $7.60(\mathrm{~s}, 1 \mathrm{H}, \mathrm{Ar}-\mathrm{H}) ; 7.30(\mathrm{~s}, 1 \mathrm{H}, \mathrm{HC}=\mathrm{N}) ; 11.90(\mathrm{~s}, 1 \mathrm{H}, \mathrm{NH}) .{ }^{13} \boldsymbol{C}-\boldsymbol{N M \boldsymbol { R }}$ (DMSO-d $\left.)_{6}\right): \delta$ ppm $23.31\left(\mathrm{CH}_{2}\right) ; 116.20(\mathrm{CN}), 120.06,122.28,123.68,127.84,129.20,129.60$, $129.80,129.80,130.00,130.02,130.02,130.02,136.80,139.10(\mathrm{C}=\mathrm{C}) ; 106.72(5$ mem ring $\mathrm{C}=\mathrm{C})$; $142.64(\mathrm{HC}=\mathrm{N}) ; 174.67(\mathrm{C}=\mathrm{O})$.

\subsubsection{2-cyano-N'-((3-(4-methoxyphenyl)-1-phenyl-1H-pyrazol-4-yl)methylene) acetohydrazide [BJ-13]}

Color-Dull yellow, Yield- 75\%, Melting Point- $233^{\circ} \mathrm{C}$, Elemental Analytical Calculation for $\boldsymbol{C}_{20} \mathrm{H}_{17} \mathrm{~N}_{5} \boldsymbol{O}_{2}(359.14 \mathrm{~g} / \mathrm{mol}): \mathrm{C}, 66.84 \%$; H, 4.77\%; N, 19.49\%; O,8.90 \%. Found: C, 66.80\%; H, 4.74\%; N, 19.45\%; O, 8.88\%; MS (m/z):359; IR (cm $\left.{ }^{-1}\right): v(\mathrm{NH}) 3010,3068 ; v(\mathrm{C}=\mathrm{N}) 1673 ; v(\mathrm{C}=\mathrm{O})$ 1716; v(N-N) 1130; v(Ar-C-H) 2820, 2962; v(Ar-C=C) $1523 ; v(\mathrm{C}=\mathrm{N}) 2328,2288 ; v(\mathrm{C}-\mathrm{O}) 1040$. ${ }^{1} \boldsymbol{H}$-NMR (DMSO-d $)$ : $\delta$ ppm $3.72\left(\mathrm{~s}, 3 \mathrm{H}, \mathrm{CH}_{3}\right) ; 4.12\left(\mathrm{~s}, 2 \mathrm{H}, \mathrm{CH}_{2}\right) ; 7.30-7.040(\mathrm{~m}, 7 \mathrm{H}, \mathrm{Ar}-\mathrm{H}) ; 7.04-$ $7.10(\mathrm{~d}, 2 \mathrm{H}, \mathrm{Ar}-\mathrm{H}) ; 8.20(\mathrm{~s}, 1 \mathrm{H}, \mathrm{HC}=\mathrm{N}) ; 8.30(\mathrm{~s}, 1 \mathrm{H}, \mathrm{HC}=\mathrm{C}) ; 12.10(\mathrm{~s}, 1 \mathrm{H}, \mathrm{NH}) .{ }^{13} \boldsymbol{C}-\boldsymbol{N M \boldsymbol { R }}$ (DMSO-d $\left.)_{6}\right): \delta$ ppm $55.96\left(\mathrm{OCH}_{3}\right) ; 24.11\left(\mathrm{CH}_{2}\right) ; 116.00(\mathrm{CN}), 114.18,114.20,116.60,120.18$, $121.24,121.60,130.18,130.20,130.20,130.20,136.66,138.34,138.40,139.16(\mathrm{C}=\mathrm{C}) ; 140.64$ $(\mathrm{HC}=\mathrm{N}) ; 114.60(\mathrm{C}-\mathrm{O}) ; 170.67(\mathrm{C}=\mathrm{O})$.

3.1.6 2-cyano-N'-((3-(4-nitrophenyl)-1-phenyl-1H-pyrazol-4-yl)methylene) acetohydrazide [BJ-14]

Color-Dark yellow, Yield- $72 \%$, Melting Point- $240{ }^{\circ} \mathrm{C}$, Elemental Analytical Calculation for $\boldsymbol{C}_{19} \mathrm{H}_{16} \mathrm{~N}_{6} \mathrm{O}_{3}(374.35 \mathrm{~g} / \mathrm{mol}): \mathrm{C}, 60.96 \%$; H, 3.77\%; N, 22.45\%; O,12.82 \%. Found: C, 60.90\%; H, $3.75 \%$; N, 22.44\%; O, 12.80\%; MS (m/z):374; IR (cm $\left.{ }^{-1}\right): v(\mathrm{NH}) 3010,3036 ; v(\mathrm{C}=\mathrm{N}) 1666$; $v(\mathrm{C}=\mathrm{O}) 1718 ; \mathrm{v}(\mathrm{N}-\mathrm{N}) 1090,1103 ; \mathrm{v}(\mathrm{Ar}-\mathrm{C}-\mathrm{H}) 2840,2910 ; \mathrm{v}(\mathrm{Ar}-\mathrm{C}=\mathrm{C}) 1528 ; \mathrm{v}(\mathrm{C}=\mathrm{N}) 2320,2280$. ${ }^{1} \boldsymbol{H}$-NMR (DMSO-d $)$ : $\boldsymbol{\delta}$ ppm $4.22\left(\mathrm{~s}, 2 \mathrm{H}, \mathrm{CH}_{2}\right) ; 7.88$ (s, 1H, Ar-H); 8.22-8.26 (d, 2H, Ar-H); 7.04$7.10(\mathrm{~m}, 7 \mathrm{H}, \mathrm{Ar}-\mathrm{H}) ; 8.20(\mathrm{~s}, 1 \mathrm{H}, \mathrm{HC}=\mathrm{N}) ; 11.90(\mathrm{~s}, 1 \mathrm{H}, \mathrm{NH}) .{ }^{13} \boldsymbol{C}$-NMR (DMSO-d $)$ : $\delta$ ppm 24.31 $\left(\mathrm{CH}_{2}\right)$; $116.09(\mathrm{CN}), 120.80,121.16,122.22,126.34,127.28,129.20,129.21,130.46,130.60$, $130.62,130.62,132.66,133.28 \mathrm{C}=\mathrm{C}) ; 139.40,139.20(\mathrm{HC}=\mathrm{N}) ; 141.30\left(\mathrm{HC}=\mathrm{NO}_{2}\right) ; 174.60(\mathrm{C}=\mathrm{O})$. 


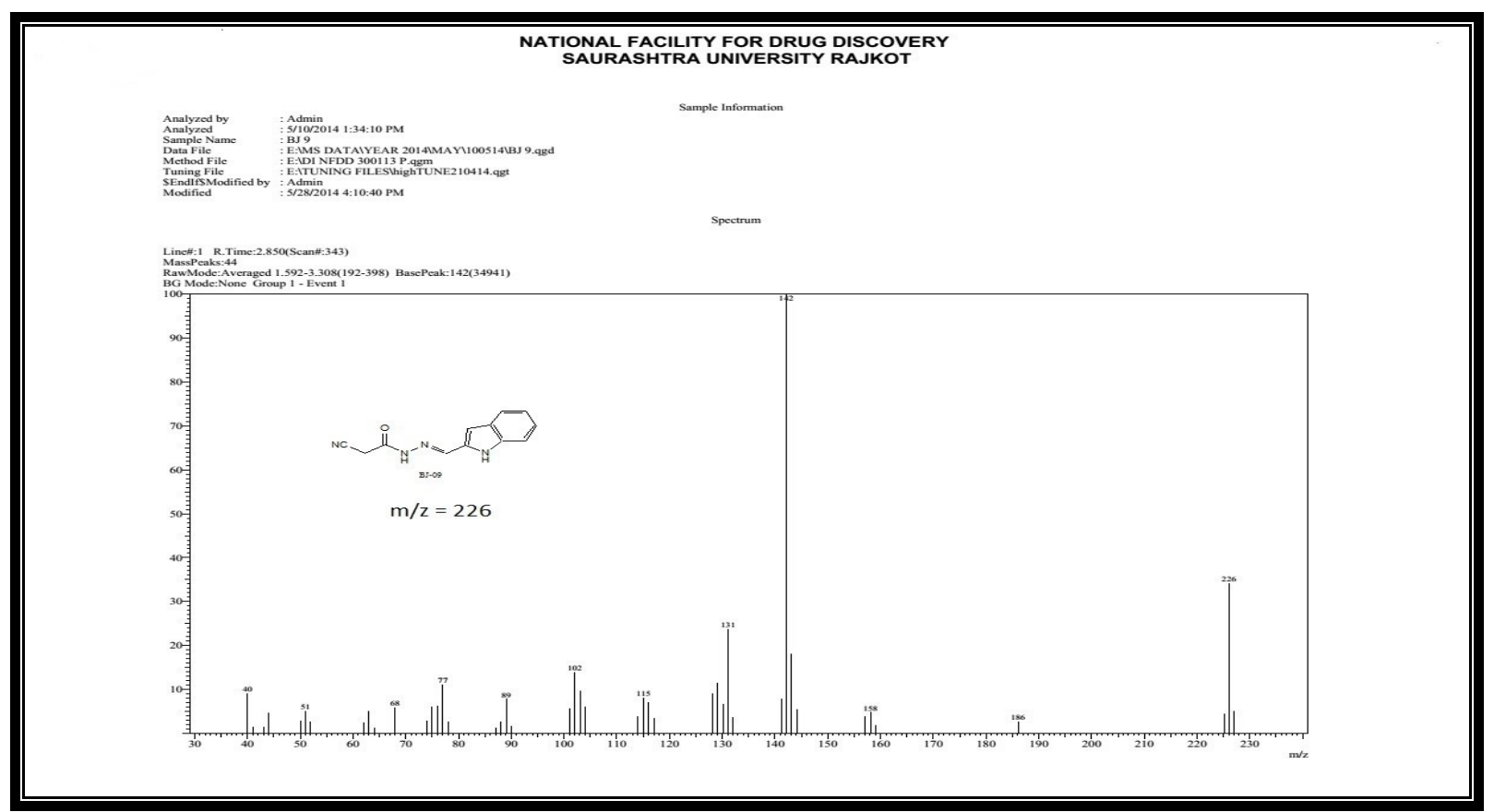

Figure. 3 Mass spectrum of BJ-09

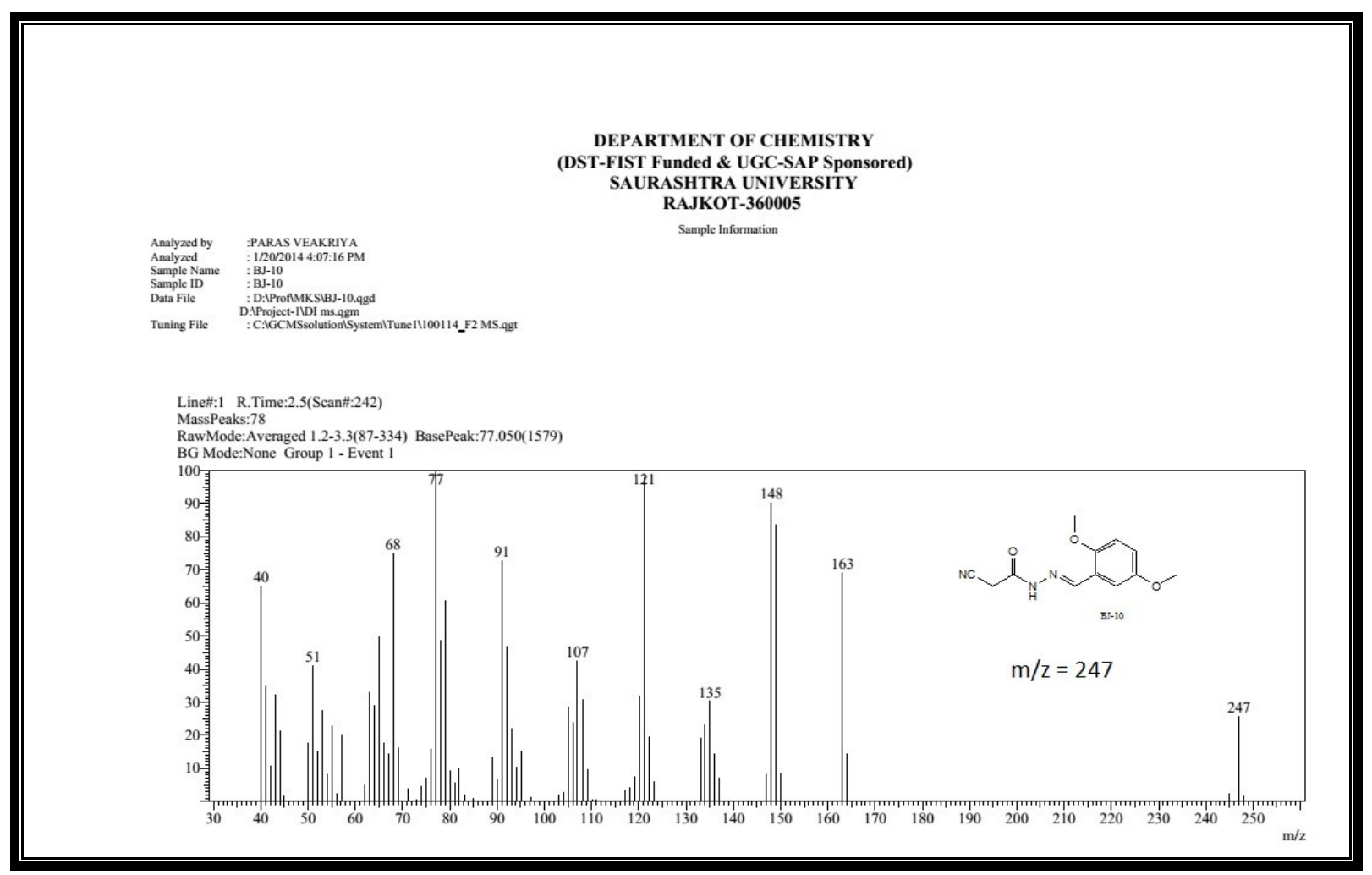

Figure. 4 Mass spectrum of BJ-10 


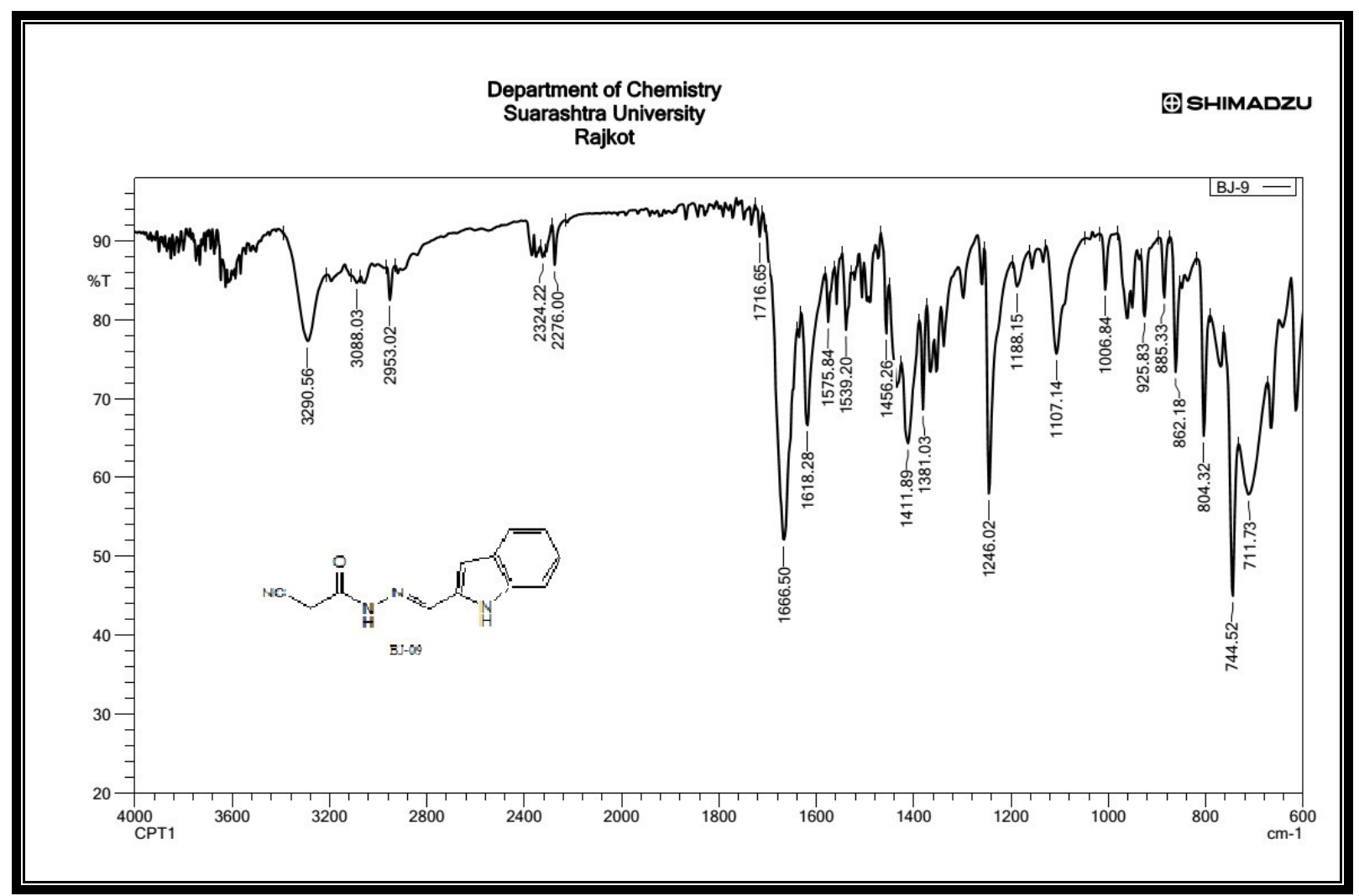

Figure. 5 IR spectrum of BJ-09

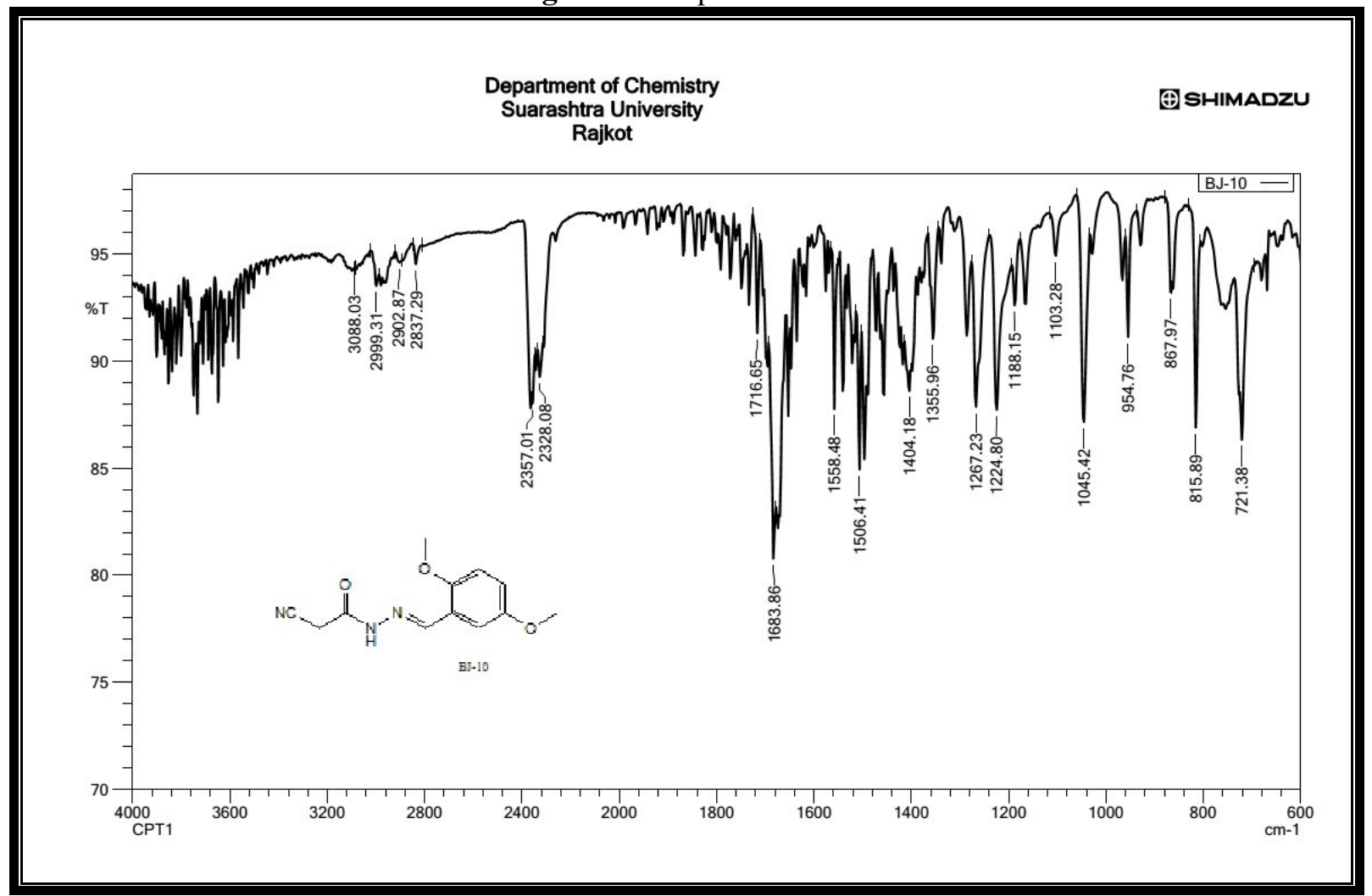

Figure. 6 IR spectrum of BJ-10 


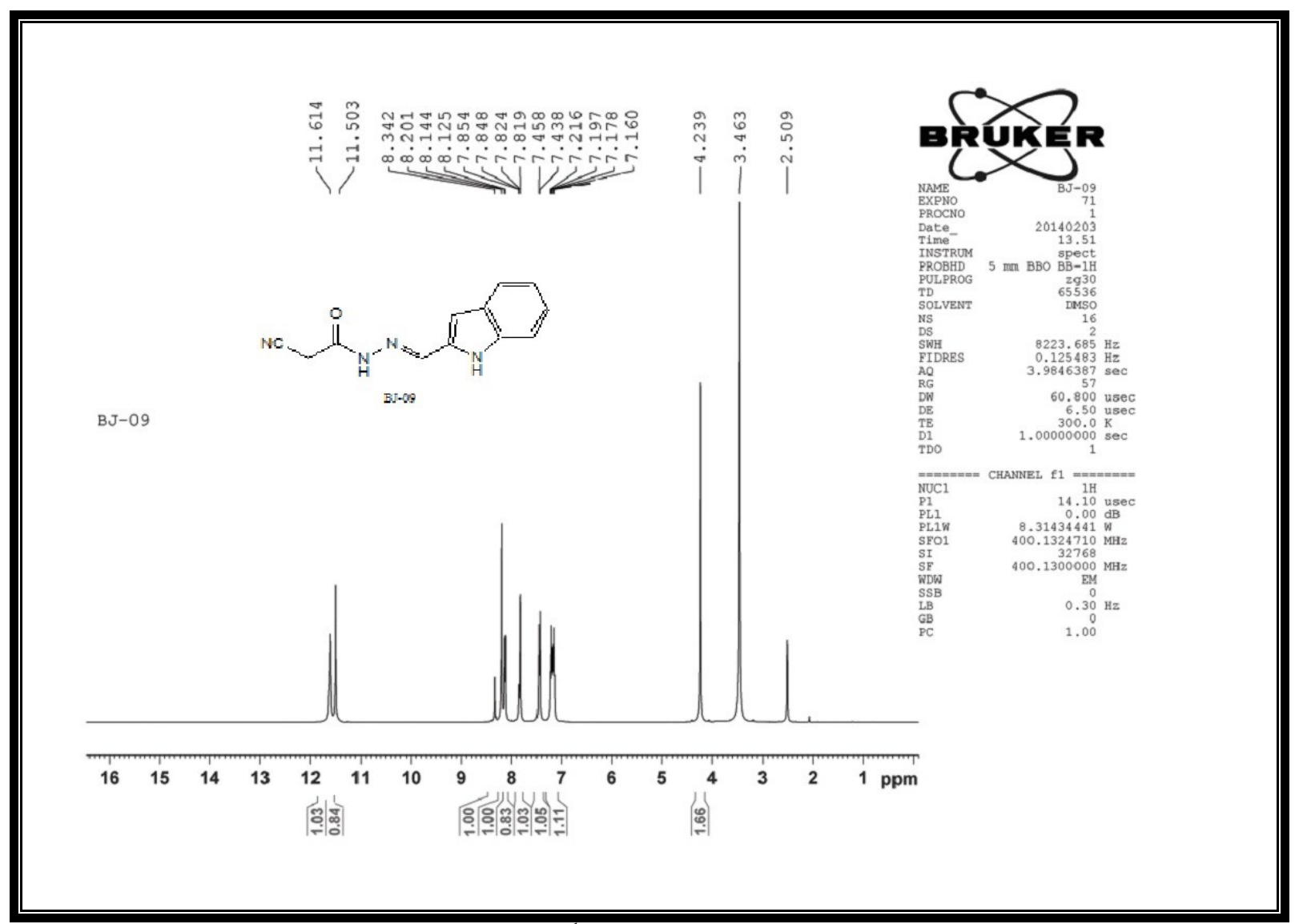

Figure. $7{ }^{1} \mathrm{H}$ NMR spectrum of BJ-09

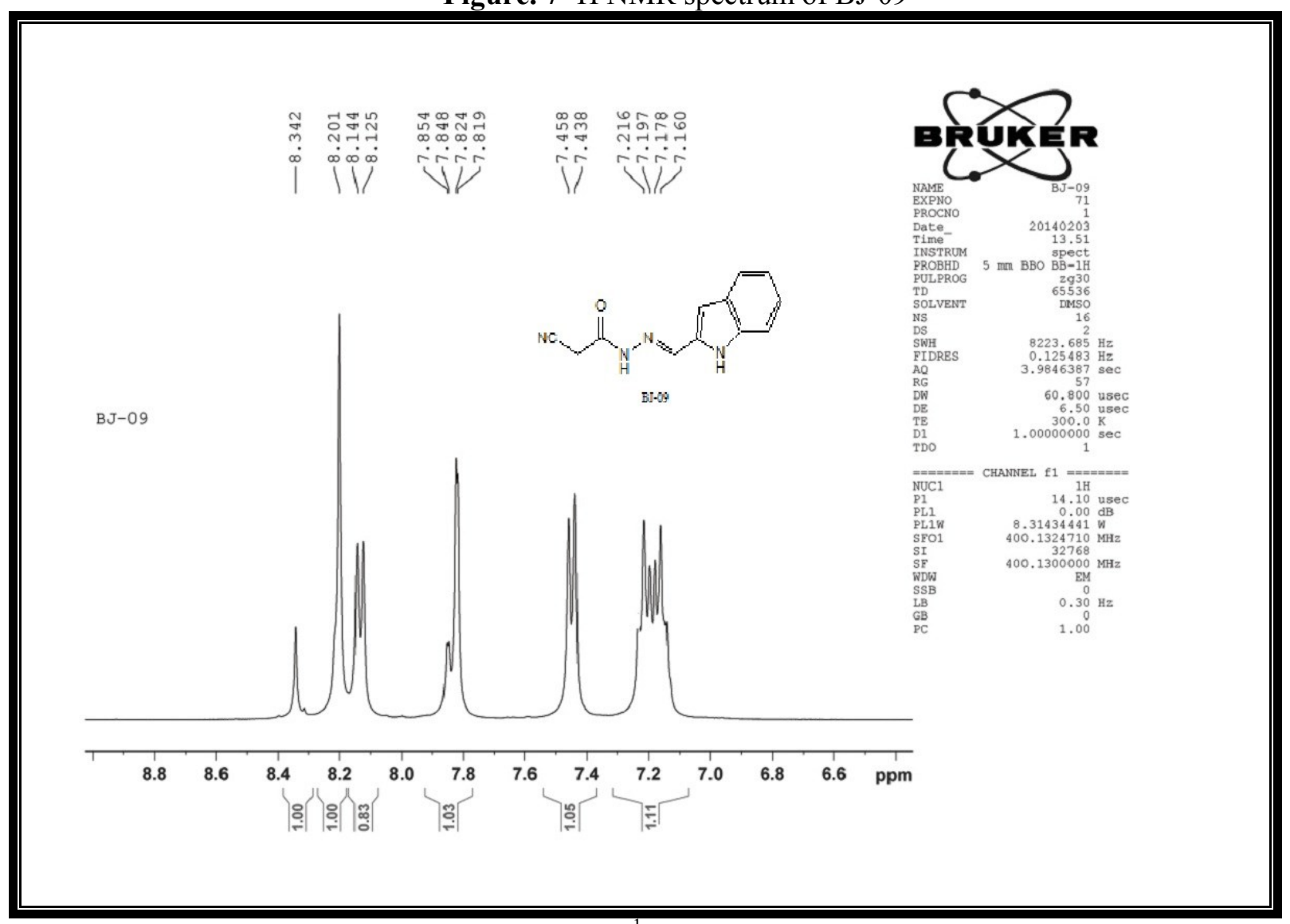

Figure. 8 Expanded ${ }^{1} \mathrm{H}$ NMR spectrum of BJ-09 


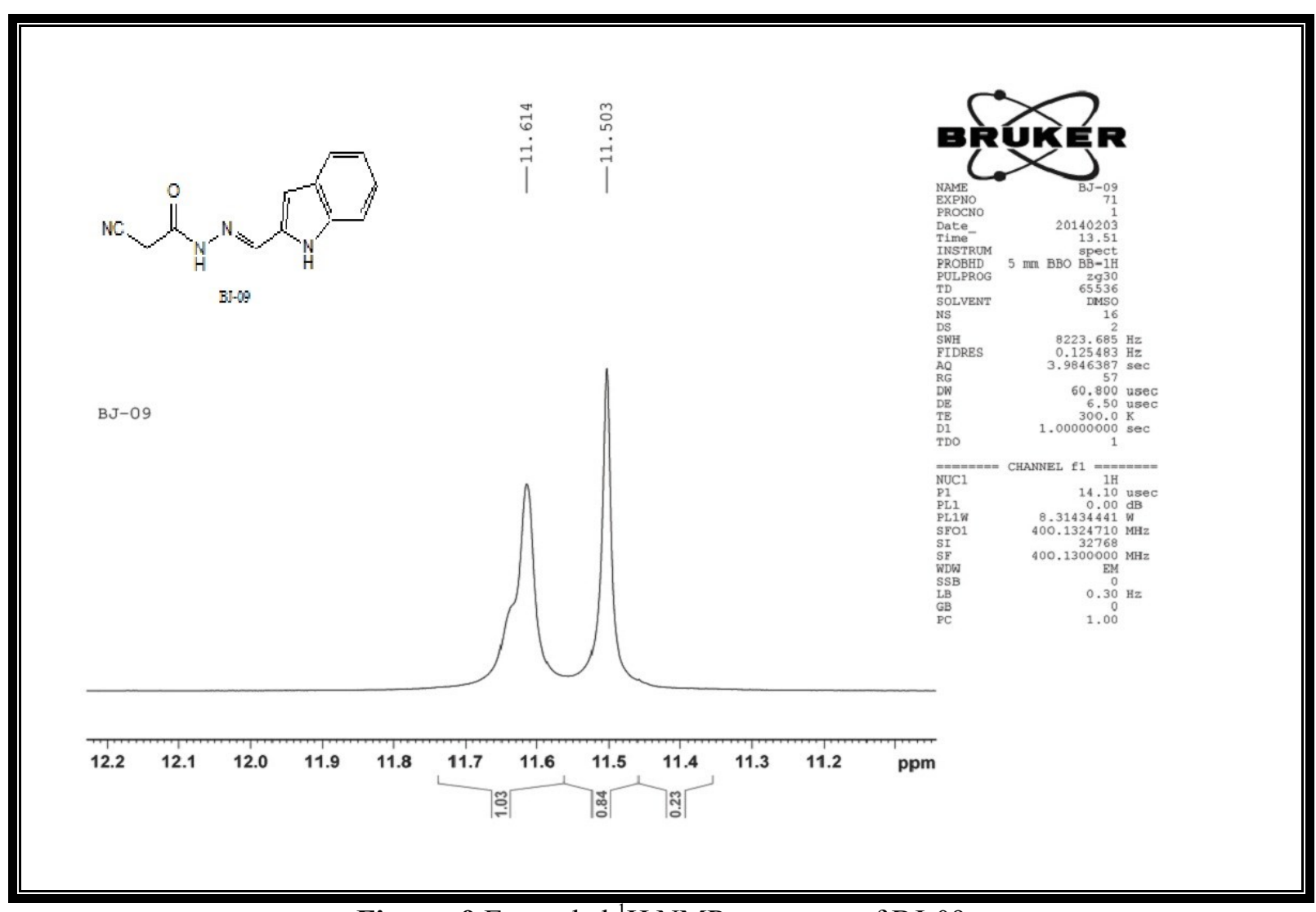

Figure. 9 Expanded ${ }^{1} \mathrm{H}$ NMR spectrum of BJ-09

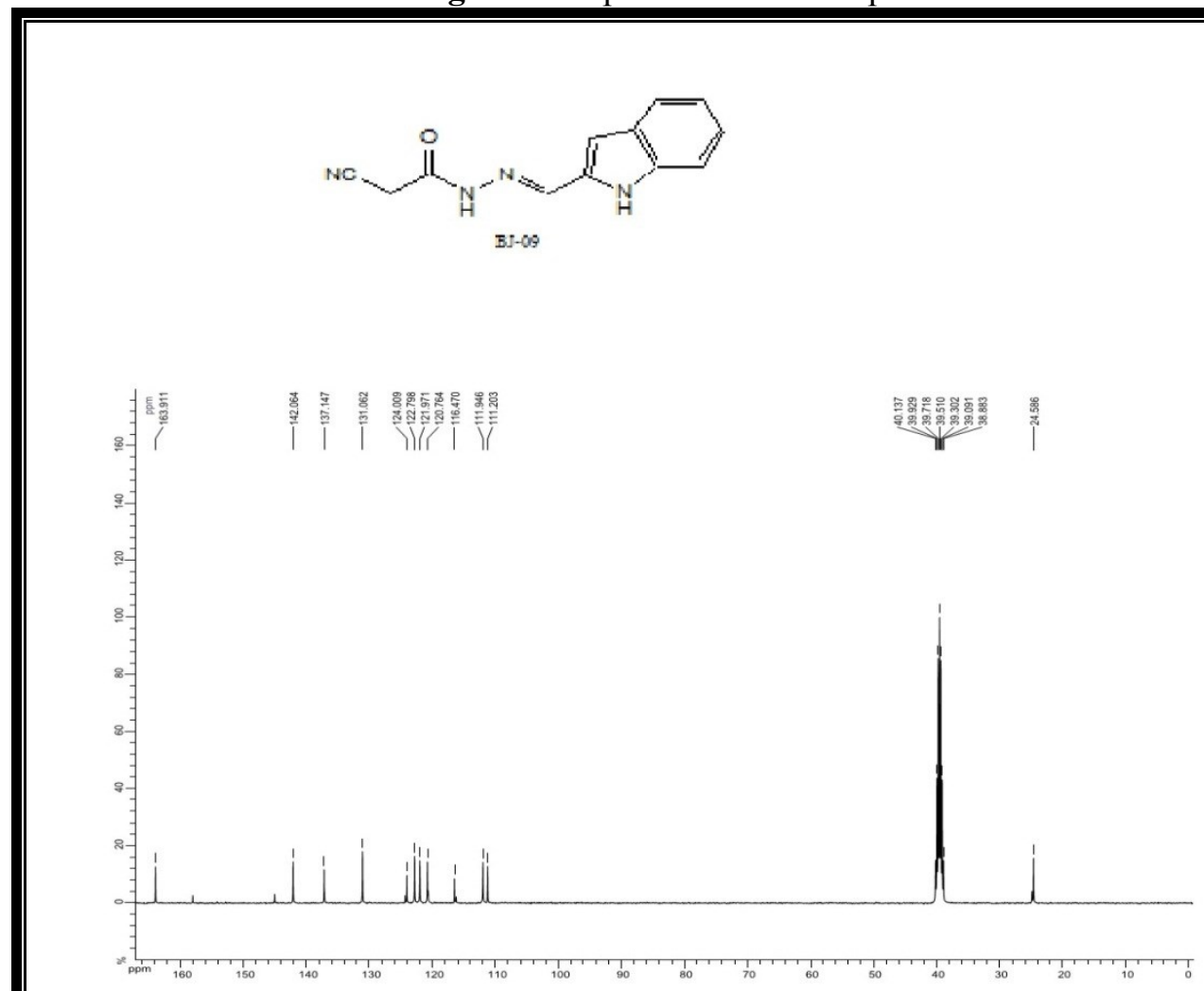

Figure. 10 ${ }^{13} \mathrm{C}$ NMR spectrum of BJ-09 


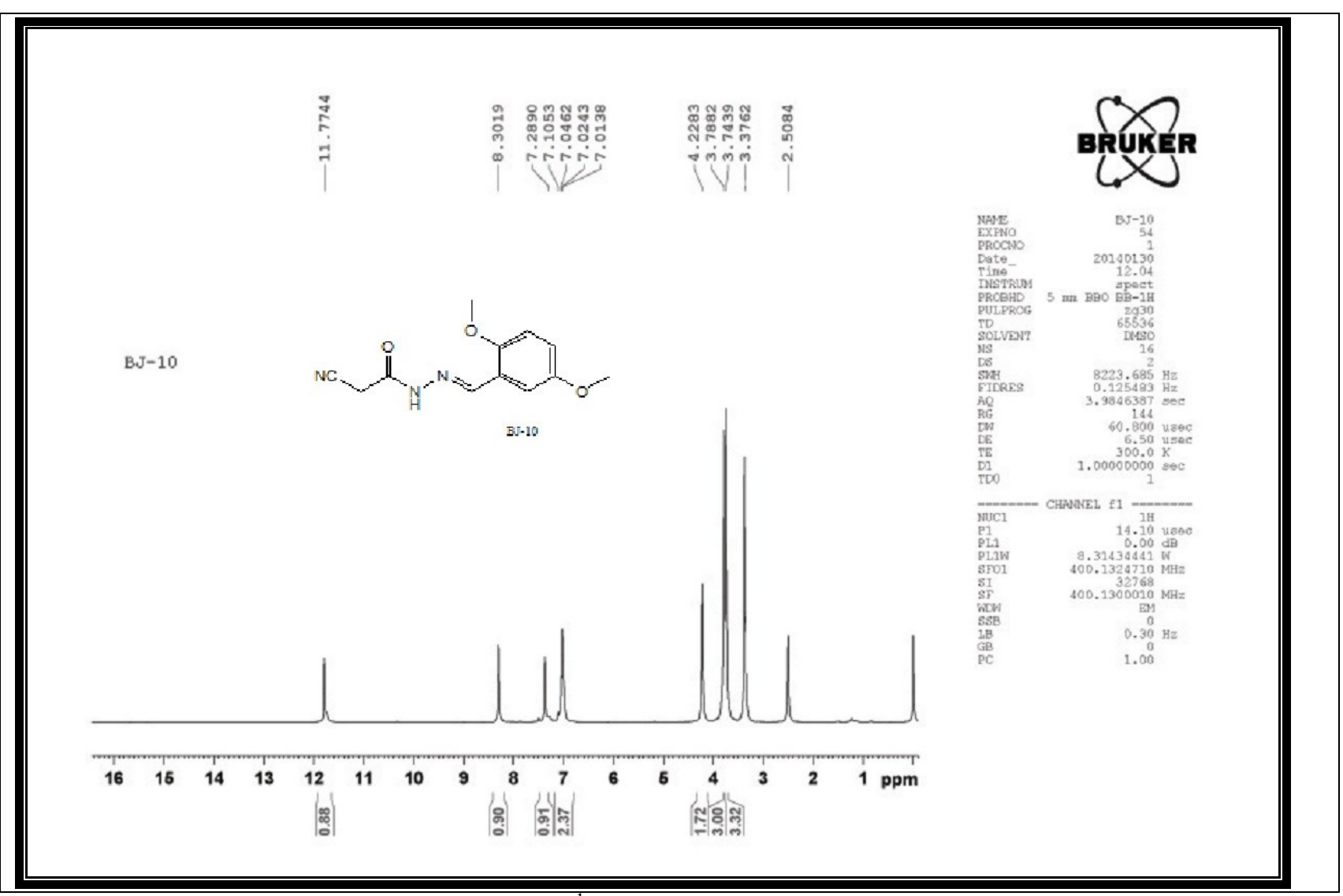

Figure. $11{ }^{\mathrm{I}} \mathrm{H}$ NMR spectrum of BJ-10

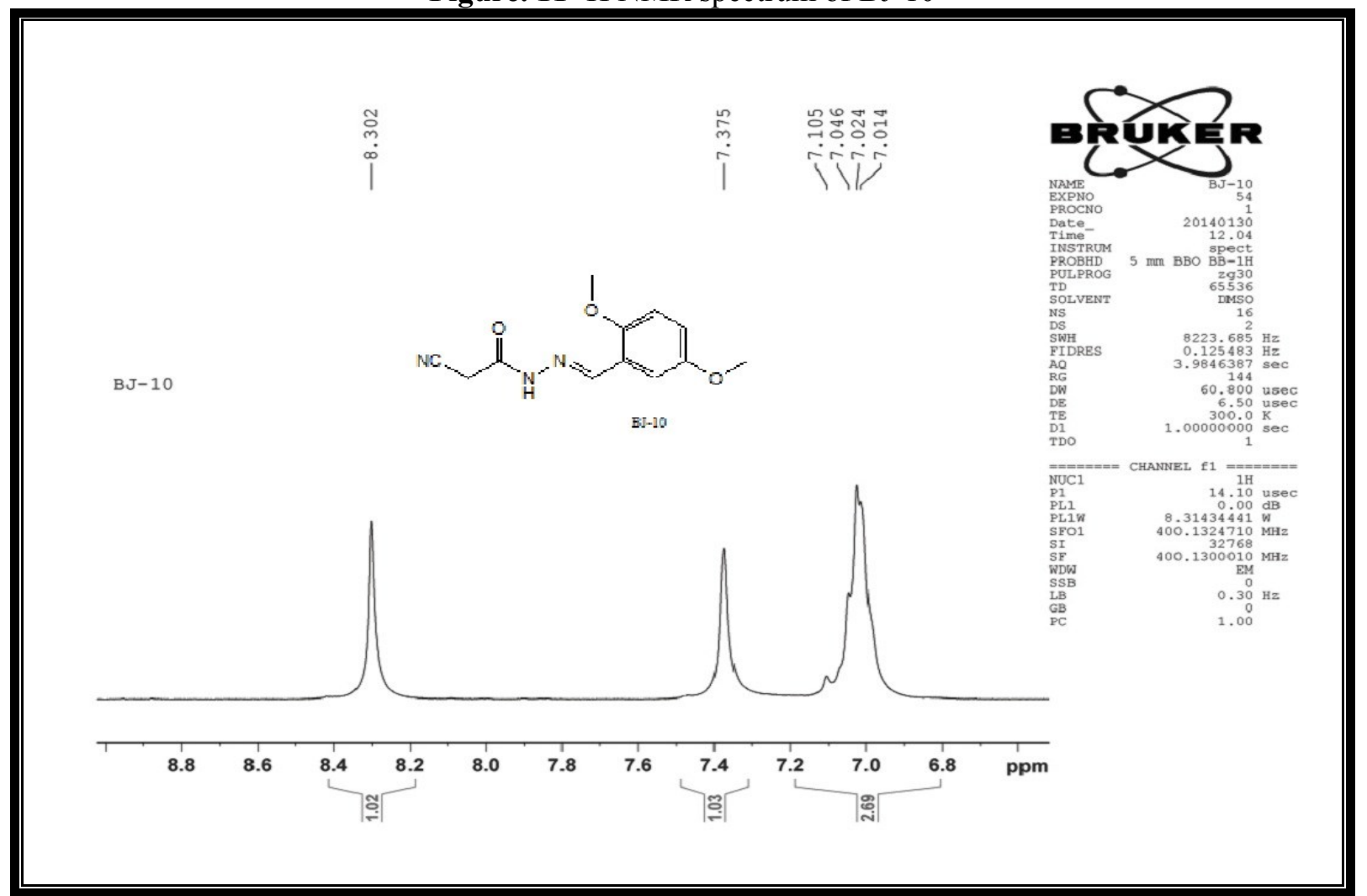

Figure. 12 Expanded ${ }^{1} \mathrm{H}$ NMR spectrum of BJ-10 


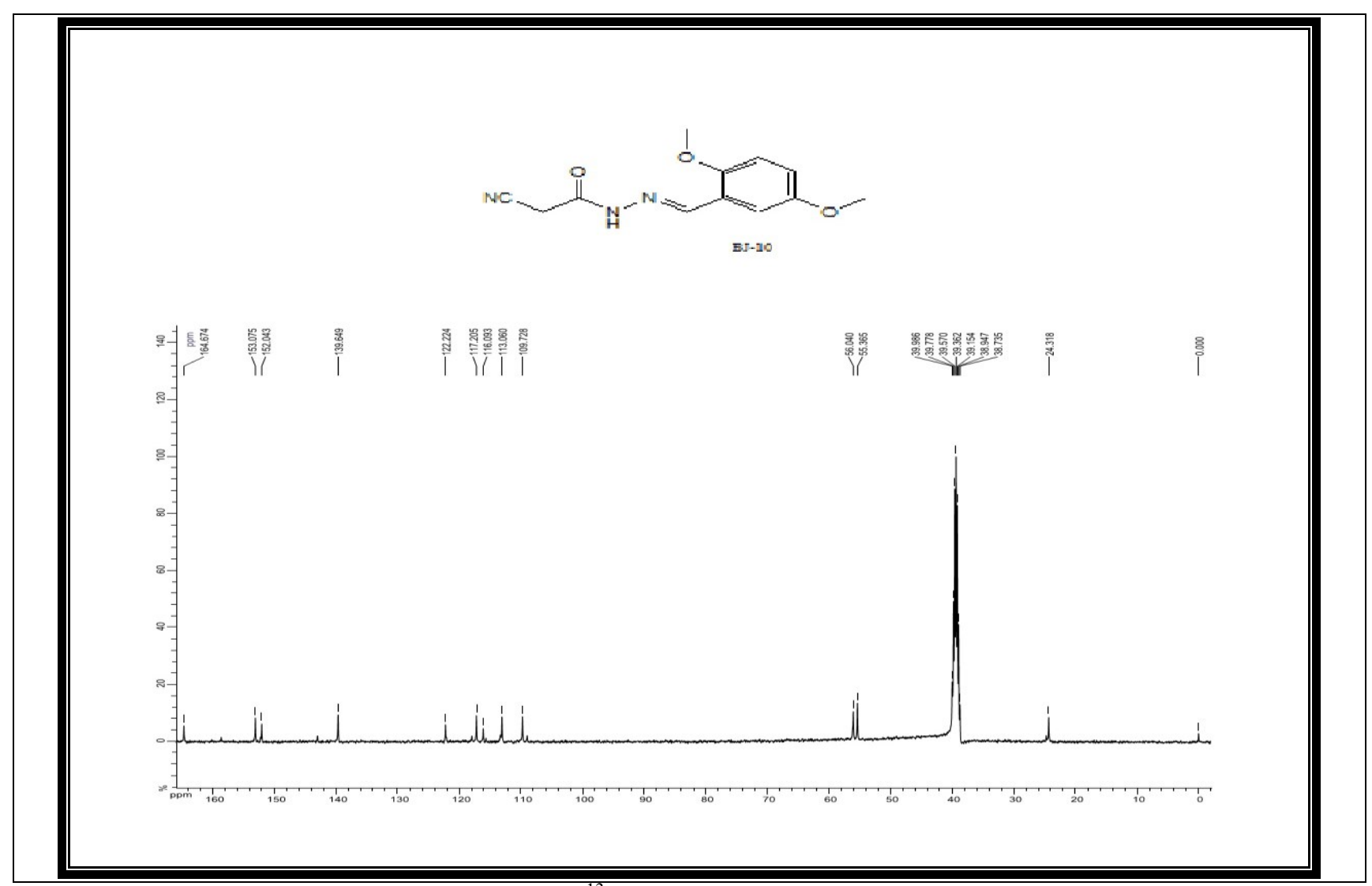

Figure. $13{ }^{13} \mathrm{C}$ NMR spectrum of BJ-10

\section{BIOLOGICAL ACTIVITY}

The above work deals with the antimicrobial screening of the compounds. The minimum inhibition concentration (MIC) values were determined in the present study.

\subsection{Antimicrobial evaluation}

The antibacterial and antifungal activity (MIC) in vitro was carried out by broth dilution method of all the compounds. Two Gram-positive bacteria Staphylococcus aureus MTCC-96, Streptococcus pyogenes MTCC 443, two Gram-negative bacteria Escherichia coli MTCC 442, Pseudomonas aeruginosa MTCC 441 and three fungal strains Candida albicans MTCC 227, Aspergillus Niger MTCC 282, Aspergillus clavatus MTCC 1323 strains were taken. As standard drugs, we have taken ampicillin, chloramphenicol, ciprofloxacin, norfloxacin, nystatin, and greseofulvin.

The minimal inhibitory concentration (MIC) values for all the newly synthesized compounds, defined as the lowest concentration of the compound preventing the visible growth, were determined by using microdilution broth method according to NCCLS standards. Serial dilutions of the test compounds and reference drugs were prepared in Muellere-Hinton agar. Drugs $(10 \mathrm{mg})$ were dissolved in dimethylsulfoxide (DMSO, $1 \mathrm{~mL})$. Further progressive dilutions with melted Muellere-Hinton agar were performed to obtain the required concentrations. In primary screening $1000 \mu \mathrm{g} \mathrm{mL}^{-1}, 500 \mu \mathrm{g} \mathrm{mL}^{-1}$ and $250 \mu \mathrm{g} \mathrm{mL}^{-1}$ concentrations of the synthesized drugs were taken. The active synthesized drugs found in this primary screening were further tested in a second set of dilution at $200 \mu \mathrm{g} \mathrm{mL}^{-1}, 100 \mu \mathrm{g} \mathrm{mL}^{-1}, 50 \mu \mathrm{g} \mathrm{mL}^{-1}, 25 \mu \mathrm{g} \mathrm{mL}^{-1}, 12.5 \mu \mathrm{g} \mathrm{mL}^{-1}$, and $6.25 \mu \mathrm{g} \mathrm{mL}^{-}$ ${ }^{1}$ concentration against all microorganisms. The tubes were inoculated with $10^{8} \mathrm{cfu} \mathrm{mL}^{-1}$ (colony forming unit $/ \mathrm{mL}$ ) and incubated at $37^{\circ} \mathrm{C}$ for $24 \mathrm{~h}$. The MIC was the lowest concentration of the tested compound that yields no visible growth (turbidity) on the plate. To ensure that the solvent had no effect on the bacterial growth, a control was performed with the test medium supplemented with DMSO at the same dilutions as used in the experiments and it was observed that DMSO had no effect on the microorganisms in the concentrations studied. 
The results obtained from antimicrobial susceptibility testing are depicted in Table 1 .

Table.1 Antibacterial and antifungal activity of synthesized compounds BJ-09 to BJ-14.

\begin{tabular}{|c|c|c|c|c|c|c|c|}
\hline \multirow[t]{3}{*}{ Code } & \multicolumn{7}{|c|}{ Minimal inhibition concentration $\left(\mu \mathrm{g} \mathrm{mL} \mathrm{L}^{-1}\right)$} \\
\hline & \multicolumn{2}{|c|}{ Gram-positive } & \multicolumn{2}{|c|}{ Gram-negative } & \multicolumn{3}{|c|}{ Fungal species } \\
\hline & S.a. & S. p. & E.c. & P.a. & C. $a$. & A. n. & A.c. \\
\hline BJ-09 & 250 & 500 & 250 & 100 & 250 & 250 & 250 \\
\hline BJ-10 & 500 & 500 & 250 & 250 & 500 & 200 & 200 \\
\hline BJ-11 & 100 & 250 & 100 & 250 & 500 & 500 & $>1000$ \\
\hline BJ-12 & 500 & 500 & 250 & 500 & 500 & $>1000$ & 500 \\
\hline BJ-13 & 250 & 62.5 & 62.5 & 500 & 250 & 500 & 250 \\
\hline BJ-14 & 250 & 100 & 250 & 125 & 500 & $>1000$ & $>1000$ \\
\hline Ampicillin & 250 & 100 & 100 & 100 & - & - & - \\
\hline $\begin{array}{l}\text { Chloram- } \\
\text { phenicol }\end{array}$ & 50 & 50 & 50 & 50 & - & - & - \\
\hline Ciprofloxacin & 50 & 50 & 25 & 25 & - & - & - \\
\hline Norfloxacin & 10 & 10 & 10 & 10 & - & - & - \\
\hline Nystatin & - & - & - & - & 100 & 100 & 100 \\
\hline Greseofulvin & - & - & - & - & 500 & 100 & 100 \\
\hline
\end{tabular}

\section{CONCLUSION}

We have synthesized Schiff base of cyanoacetohydrazide and different substituted aromatic aldehyde under acidic condition and at room temperature. All synthesized Schiff base were obtained in good to moderate yield. All synthesized Schiff base were characterized by Mass spectrometry, IR, ${ }^{1} \mathrm{H}$ and ${ }^{13} \mathrm{C}$ NMR and Elemental Analysis. They are incorporated with the structure of compounds. All the compounds are showing moderate antibacterial with compare to standard Drugs and are less active towards fungal strains.

\section{ACKNOWLEDGMENT}

The Authors are very thankful to Department of Chemistry, Saurashtra University, Rajkot for providing facilities and NFDD Center (National Facilities for Drugs Discovery) Rajkot for NMR analysis.

\section{References}

[1] S. Rallas, N. Gulerman, H. Erdeniz, Farmaco 57 (2002) 171-174.

[2] A. Gursoy, N. Terzioglu, G. Otuk, Eur. J. Med. Chem. 32 (1997) 753-757.

[3] C. Walsh, Nature 406 (2000) 775-778.

[4] S. Rollas, N. Kalyoncuoğlu, D. Sur-Altiner and Y. Ye- ğenoğlu, Pharmazie, Vol. 48, No. 4, (1993), 308-309.

[5] S. Papakonstantinou-Garoufalias, N. Pouli, P.-Marakos and A. Chytyro-Glouladas, Farmaco, Vol. 57, No. 12, (2002), pp. 973-977.

[6] M. Kidwai, R. Kumar, A. Srivastava and H. P. Gupta, Bioorganic Chemistry, Vol. 26, No. 5, (1998), pp. 289-294.

[7] V. V. Kachhadia, M. R. Patel and H. S. Joshi, Journal of the Serbian Chemical Society, Vol. 70, No. 2, (2005), pp. 153-161.

[8] P. K. KadabaCurrent Medicinal Chemistry, Vol. 10, No. 20, (2003), pp. 2081-2108. 
[9] İ. küçükgüzel, Ş. G. Küçükgüzel, S. Rollas, G. Ö.-Saniş, O. Özdemir, I. Bayrak, T. Altuğand J. P. Stables, Farmaco, Vol. 59, No. 11, (2004), pp. 893-901.

[10] M. D. Mullican, M. W. Wilson, D. T. Connor, C. R. Kostlan, D. J. Schrier and R. D. Dyer, Journal of Medicinal Chemistry, Vol. 36, No. 8, (1993), pp. 1090-1099.

[11] E. Palaska, G. Şahin, P. Kelicen, N. T. Durlu and G. Altinok, Farmaco, Vol. 57, No. 2, (2002), pp. 101-107.

[12] A.Varvaresou, T. Siatra-Papastaikoudi, A. Tsotinis, A. TsantiliKakoulidou and A. Vamvakides, Farmaco, Vol. 53, No. 5, (1989), pp. 320-326.

[13] B. S. Holla, B. Veerendra, M. K. Shivanada and B. Poo- jary, european Journal of Medicinal Chemistry, Vol. 38, No. 7-8, (2003), pp. 759-767.

[14] J. H. Lange, H. H. Stuivenberg, H. K. Coolen, T. J. Adolfs, A. C. McCreary, H.G. Keizer, H. C. Wals, W.Veerman, A. J. Borst, W. de Looff, P. C. Verveer and C. G. Kruse, Journal of Medicinal Chemistry, Vol. 48, No. 6, (2005), pp. 1823-1838.

[15] N. Szuber, J.L. Buss, S.S. Lin, H. Felfly, M. Trudel, P. Ponka, Exp. Hematol. 36(2008) 773785. 